\title{
THE ROLE OF MATERIAL NON-HOMOGENEITIES ON THE FORMATION AND EVOLUTION OF STRAIN NON-UNIFORMITIES IN THERMOVISCOPLASTIC SHEARING
}

\author{
BY \\ THEOCHARIS BAXEVANIS AND NICOLAS CHARALAMBAKIS \\ Department of Civil Engineering, Aristotle University, GR 54006 Thessaloniki, Greece
}

\begin{abstract}
In this paper we present the effect of macroscopic non-homogeneities on the distribution and evolution of strain non-uniformities during the shearing of thermoviscoplastic materials. The thermomechanical parameters (strain hardening, strain rate sensitivity, and thermal softening), as well as all of the material parameters are supposed to depend explicitly on the space variable. We show that, even under stability conditions, the strain exhibits intense, time-increasing non-uniformities, following the non-homogeneities, in a specific rate, which depends on the degree of non-homogeneity exhibited by the thermomechanical parameters. By considering both the isothermal and anisothermal cases, we obtain results indicating that non-uniformity measures, based on the control of the strain gradient, are more suitable to give stability conditions of nonhomogeneous materials. Moreover, we present numerical results concerning the interplay between material non-homogeneities, initial defects, and boundary conditions for two specific cases: the shearing of a reinforced slab and the shearing of a plate composed of several thin layers of periodic structure. The results are in complete agreement with the analytical behavior of the model.
\end{abstract}

1. Introduction. During plastic deformation of metals, the strength of the material is decreasing with respect to temperature (or strain, in isothermal models, where thermal effects are implicitly taken into account). Then, if the temperature and/or strain are highly non-uniform, narrow zones of weaker material appear, especially under high strain rate. Initial strain non-uniformities, due to geometrical defects, may cause additional weakness. The localized shear deformation may lead to the emergence of adiabatic shear bands and, eventually, to catastrophic fracture.

Received January 17, 2002.

2000 Mathematics Subject Classification. Primary 35B30, 35B40, 74D10.

E-mail address: charalam@civil.auth.gr 
In this paper, we present the effect of material inhomogeneities on the emergence and evolution of strain non-uniformities during the simple shearing of softened ratedependent materials. We are interested on macroscopic inhomogeneities, in the sense that the material properties, and more specifically the thermomechanical functions, depend explicitly on the space variable.

The question of strain localization is related to the study of the large time behavior of strain or strain gradient. An efficient mathematical way, introduced by Dafermos (1982, 1985), Dafermos and Hsiao (1983), and Tzavaras (1986, 1987, 1991, 1992), is based on the application of nonlinear analysis techniques, in order to obtain stability and instability conditions between the thermomechanical parameters, namely the thermal softening, the strain hardening, and the strain rate sensitivity (see also Charalambakis $(1984,1985)$, Chen et al. (1989), Maddoks and Malek-Madani (1992), Liu and Jiang (2003) and, for the two-dimensional case, Rigatos and Charalambakis (2001)). More specifically, Tzavaras presented a consistent existence and continuation theory for the model of ratedependent softened materials. According to this, if the initial data are smooth and positive, then there exists a unique classical solution, defined on a maximal time interval of existence, which could be infinite or finite. In the latter case, the shearing under prescribed tractions at the boundaries leads to a strain and strain-rate blow-up, while the stress remains bounded. In this context, the concept of $L_{\infty}$ - localization was used by Molinari and Clifton (1987), giving similar results. This concept may provide a measure of strain non-uniformities by following the ratio of strains at two material points. These findings agree with the theoretical and experimental work of Clifton et al. (1984), Wright and Batra (1985), Wright and Walter (1987), and Shawki and Clifton (1989). Moreover, it is of interest to study the implications of some other additional functions, such as the cold work, which is the portion of plastic work stored to create a new situation of crystal defects (see Hodowany et al. (2000), Rosakis et al. (2000), and Charalambakis (2001)). It seems that the variation of this quantity, with respect to strain and strain-rate, is important for the understanding of the instability regime.

However, in the case of homogeneous materials, these techniques lead to very large values of critical time, at which instability is manifested as catastrophic failure. This is due to the fact that, in this case, the concept of shear banding is related to the blow-up of solutions of the system of partial differential equations describing the problem, i.e., to extremely large values of strain or temperature, while, in the reality, only a finite value of strain, called critical strain, suffices to produce shear banding. This is the reason for the elaboration of some more engineering localization measures, based on linearized analysis techniques, by Bai (1982), Burns (1983), Molinari (1985), Dodd and Bai (1987), Fressengeas and Molinari (1987), and Leroy and Molinari (1992). Some of these methods control the evolution of the relative perturbation in order to obtain critical conditions of the process.

In this paper, we assume that the flow stress of a power law material, exhibiting softening, depends explicitly on the space variable $x$. We are concerned with two specific types of flow stress, namely (see Shawki and Clifton (1989) and Tzavaras (1992) for the 
homogeneous case)

$$
\sigma(x, t)=G(x) \theta(x, t)^{-a(x)} \gamma(x, t)^{m(x)} \gamma_{t}(x, t)^{n(x)}, \quad m(x)<a(x), \quad \forall x,
$$

for the anisothermal case, and

$$
\sigma(x, t)=G(x) \gamma(x, t)^{-m(x)} \gamma_{t}(x, t)^{n(x)}, \quad m(x)<\frac{n(x)}{3}<\frac{1}{3}, \quad \forall x,
$$

for the isothermal. In (1) and (2), $\theta, \gamma$, and $\gamma_{t}$ denote the temperature, strain, and strain rate field, respectively (all subscripts indicating differentiation with respect to the variable indicated), while $G(x)$ is a material parameter. The isothermal case (2) is justified by the assumption that the temperature acts as a hidden variable, while the destabilization is driven by the strain softening. In (1) and (2), the functions $a(x), m(x)$, and $n(x)$ respectively denote the thermal softening, the strain hardening (1) or strain softening (2), and the strain rate sensitivity, all supposed to be space dependent. We note that $a(x), m(x)$, and $n(x)$ are supposed to be functions that smoothly approximate the nonhomogeneous thermomechanical parameters of the material. We also note that the restriction in (2), between strain softening and strain-rate sensitivity functions, is only technical.

In Sec. 2, we first verify that similar stability and instability criteria with the homogeneous case are valid, with the thermal softening $a(x)$ in (1) (or the strain softening $m(x)$ in (2)) being the leading factor of instability. We next show the main result of this paper by following the evolution in time of the strain gradient under stability conditions. Namely, we show that, even in the case of uniform initial straining, the strain gradient increases in a strongly non-linear way with time in material regions where the thermomechanical parameters vary rapidly. Then, it appears that the strain gradient distribution, for large values of time, exhibits an intense oscillation in space, looking like a function which converges weakly, as the scale parameter tends to zero (Bensoussan et al. (1978), Suquet (1982)). This result seems to extend the weak formulation results of a thermoviscoplastic stratified material without strain hardening, presented in Charalambakis and Murat (1989 and 1990), where it was shown that the temperature field converges in a weak manner as the thickness of the layers tends to zero, while the stress converges strongly. Moreover, in the special case of quasi-static approximation, we show that the material inhomogeneities, combined with initial temperature or initial strain non-uniformities sufficiently large, result to $L_{\infty}$-localization, even in the case of stability conditions between the thermomechanical parameters.

Finally, in Sec. 3 we present numerical results confirming the above findings. More specifically, we show the interplay between the destabilizing role of boundary shear forces, initial geometrical defects, and material inhomogeneities in two specific cases of nonhomogeneous ductile materials, namely a material reinforced by two thin metallic plates with nicer thermomechanical parameters and a stratified slab composed of several thin layers of periodic structure. In both cases, the relative variation $(m(x)-a(x)) / n(x)$ (for the anisothermal case) or $m(x) / n(x)$ (for the isothermal case) seems to be the leading factor for the localization of the strain gradient in material regions, where these parameters vary rapidly. 
2. The Main Results. We consider the simple shearing of a plate, made by a nonhomogeneous material, between the planes $x=0$ and $x=h$, in the $y$-direction, perpendicular to $x$. The shearing is uniform in $y$ and $z$ and is caused by steady shear forces or velocities at the boundaries. Then, the process is described by the following initialboundary system of non-linear partial differential equations for $(x, t) \in(0, h) \times(0, T)$ :

$$
\begin{gathered}
\rho(x) \nu_{t}(x, t)=\sigma_{x}(x, t), \\
c(x) \rho(x) \theta_{t}(x, t)=\beta\left(x, \gamma(x, t), \theta(x, t), \gamma_{t}(x, t)\right) \sigma(x, t) \nu_{x}(x, t), \\
\gamma_{t}(x, t)=\nu_{x}(x, t), \\
\sigma(0, t)=\sigma(h, t)=S \quad \text { or } \quad \nu(0, t)=0, \quad \nu(h, t)=V, \\
\nu(x, 0)=\nu_{0}(x) \quad \text { with } \quad \nu_{x}(x, 0)>0, \quad \theta(x, 0)=\theta_{0}(x)>0, \quad \gamma(x, 0)=\gamma_{0}(x)>0,
\end{gathered}
$$

where $\sigma$ is given by (1) or (2). We note that in the isothermal case (2), Eq. (4) decouples from (3) and (5). We recall that $\nu, \theta, \gamma$, and $\sigma$ denote the velocity, temperature, strain, and stress field, respectively. In the above system, $\rho$ and $c$ are the density and specific heat, respectively, while the function $\beta$ denotes the part of the plastic energy converted into heat (Hodowany et al. (2000) and Rosakis et al. (2000)). The existence and large time behavior of solutions of the uniform shearing for the case of a homogeneous material with constant $\beta$ was fully analyzed by Tzavaras $(1987,1991,1992)$, where stability and instability conditions were presented. The same conditions are valid for the case of a material with $\beta$ dependent on temperature, strain, and strain-rate, provided that some additional restrictions on $\beta$ hold (Charalambakis (2001)).

We first study the stability conditions of the above system (3)-(7) and (1) or (2). By applying the technique proposed by Tzavaras $(1991,1992)$, we differentiate (1) with respect to time and use (3) and (5) to find

$$
\begin{aligned}
L_{1}[\sigma] & =\left(\sigma(x, t)^{1 / n(x)}\right)_{t}-G(x)^{1 / n(x)} \theta(x, t)^{-a(x) / n(x)} \gamma(x, t)^{m(x) / n(x)} \\
& \cdot\left(\rho(x)^{-1} \sigma_{x x}(x, t)+(1 / \rho(x))_{x} \sigma_{x}(x, t)\right) \\
& -(1 / n(x)) G(x)^{-1 / n(x)} \theta(x, t)^{a(x) / n(x)} \gamma(x, t)^{-m(x) / n(x)} \sigma(x, t)^{2 / n(x)} \\
& \cdot\left(-a(x) \rho(x)^{-1} c(x)^{-1} \beta\left(x, \gamma(x, t), \gamma_{t}(x, t), \theta(x, t)\right) \sigma(x, t) \theta(x, t)^{-1}+m(x) \gamma(x, t)^{-1}\right) \\
& =0
\end{aligned}
$$

under boundary conditions (6, first) or (see (6, second) and (3))

$$
\sigma_{x}(0, t)=\sigma_{x}(h, t)=0,
$$

and initial conditions defined by (7) and (1). By applying comparison theorems for the above problem (see Appendix 5) we find that the shearing (3), (4), (5), and (1) is stable for $T \rightarrow \infty$ under boundary conditions (6, first) and (6, second) provided that, for $x \in(0, h)$,

$$
p(x) \equiv m(x)+n(x)-a(x)>0,
$$


and

$$
\sup _{\substack{x \in[0, h] \\ t \in[0, T]}}\left(\beta^{*}(x, t) c(x)^{-1} \rho(x)^{-1}\right)<\inf _{x \in[0, h]}\left(a(x) m(x)^{-1}\right) \inf _{\substack{x \in[0, h] \\ t \in[0, T]}}\left(\beta^{*}(x, t) c(x)^{-1} \rho(x)^{-1}\right),
$$

where $\beta^{*} \equiv \beta\left(x, \gamma(x, t) \gamma_{t}(x, t), \theta(x, t)\right)$. We note that this function is supposed to be a given smooth function, which can be defined by studying experimentally the dependence of $\beta$ on $\gamma, \gamma_{t}$, and $\theta$ in each material component. The above condition (10) is similar to the condition presented by Tzavaras (1992), while (11) is analogous to the condition presented by Charalambakis (2001). However, as we will see below, condition (10) cannot prevent the development of highly non-uniform strain at the boundaries of non-homogeneities.

We now start from the isothermal case (2) and, following the same steps, we differentiate (2) with respect to time and use (3) and (5) to obtain

$$
\begin{array}{r}
L_{2}[\sigma]=\sigma_{t}(x, t)-n(x) G(x)^{1 / n(x)} \sigma^{(n(x)-1) / n(x)} \gamma(x, t)^{-m(x) / n(x)} . \\
\left(\rho(x)^{-1} \sigma_{x}(x, t)\right)_{x}+m(x) G(x)^{-1 / n(x)} \sigma^{(1+n(x)) / n(x)} \gamma(x, t)^{(m(x)-n(x)) / n(x)},
\end{array}
$$

under boundary conditions (6, first) or (9). Applying the comparison theorem (see Appendix 6), we find that

$$
\sigma(x, t) \leq \sup _{x \in[0, h]} \sigma_{0}(x)
$$

Using (2) and integrating over $t$,

$$
\begin{aligned}
& \gamma(x, t)^{(n(x)-m(x)) / n(x)}-\gamma_{0}(x)^{(n(x)-m(x)) / n(x)} \\
& =((n(x)-m(x)) / n(x)) G(x)^{-1 / n(x)} \int_{0}^{t} \sigma(x, t)^{1 / n(x)} \mathrm{d} \tau
\end{aligned}
$$

from which, recalling (13), we conclude that the process $(3),(5),(1)$ is stable for $T \rightarrow \infty$, provided that

$$
n(x)-m(x)>0, \quad \forall \quad x \in[0, h] .
$$

However, this condition cannot stop the monotone increase with time of strain nonuniformities at the interfaces of different material components. To see that, we must follow the large time behavior of the strain gradient, derived from (14). A simple calculation gives, with $\omega(x) \equiv m(x) / n(x)$,

$$
\begin{aligned}
& (1-\omega(x)) \gamma(x, t)^{-\omega(x)} \gamma_{x}(x, t)=\omega_{x}(x) \log (\gamma(x, t)) \gamma(x, t)^{1-\omega(x)}+\left(\gamma_{0}(x)^{1-\omega(x)}\right)_{x} \\
& -G(x)^{-1 / n(x)}\left(\log (G(x))(1-\omega(x))(1 / n(x))_{x}+\omega_{x}(x)\right) \int_{0}^{t} \sigma(x, t)^{1 / n(x)} \mathrm{d} \tau \\
& +(1-\omega(x)) G(x)^{-1 / n(x)}(1 / n(x))_{x} \int_{0}^{t} \log (\sigma(x, \tau)) \sigma(x, \tau)^{1 / n(x)} \mathrm{d} \tau \\
& +(1-\omega(x)) G(x)^{-1 / n(x)}(1 / n(x)) \int_{0}^{t} \sigma(x, \tau)^{1 / n(x)-1} \sigma_{x}(x, \tau) \mathrm{d} \tau \\
& -(1-\omega(x)) G(x)^{1 / n(x)-1} G_{x}(x)(1 / n(x)) \int_{0}^{t} \sigma(x, \tau)^{1 / n(x)} \mathrm{d} \tau
\end{aligned}
$$


Using the large time behavior of $\gamma(x, t)$ and $\sigma(x, t)$ (see (B.37) and (B.38)), we deduce from (16)

$$
\begin{aligned}
& \gamma(x, t)^{-\omega(x)} \gamma_{x}(x, t) \sim t^{(2-\omega(x)) /(1-\omega(x))} \omega_{x}(x) \\
& +t \cdot\left((\omega(x)-1)\left((1 / n(x))_{x}-1 / n(x)\right)-\omega_{x}(x)\right) \\
& +(1-\omega(x))(1 / n(x)) \int_{0}^{t} \sigma(x, \tau)^{1 / n(x)-1} \sigma_{x}(x, \tau) \mathrm{d} \tau .
\end{aligned}
$$

Recalling (B.46) and (B.52) of Appendix 6, we verify that, since

$$
\begin{aligned}
\int_{0}^{t} \sigma(x, \tau)^{1 / n(x)-1} \sigma_{x}(x, \tau) \mathrm{d} \tau & \leq \frac{1}{2} \int_{0}^{t} \sigma(x, \tau)^{2 / n(x)-2} \mathrm{~d} \tau+\frac{1}{2} \int_{0}^{t} \sigma_{x}(x, \tau)^{2} \mathrm{~d} \tau \\
& \leq K t+K \int_{0}^{t} \int_{0}^{h} \nu_{x t}(x, \tau)^{2} \mathrm{~d} x \mathrm{~d} \tau
\end{aligned}
$$

the integral in the right side of (17) is bounded by $K t$, since $m<n-m$. $K$ denotes a generic constant which can be estimated in terms of the initial data and the properties of the given functions of the problem. Therefore, there exists a sufficiently large time, after which

$$
\gamma(x, t)^{-\omega(x)} \gamma_{x}(x, t) \sim K t^{(2-\omega(x)) /(1-\omega(x))} \omega_{x}(x),
$$

or, by (B.38),

$$
\gamma_{x}(x, t) \sim K t^{2 /(1-\omega(x))} \omega_{x}(x) .
$$

We conclude from (19) that the material non-homogeneities, expressed by the slope of $\omega(x)=m(x) / n(x)$, cause increasing strain non-uniformities with a rate which also depends on $\omega$ : the larger the slope $\omega_{x}$ of the ratio of the strain softening $m$ by the strain rate sensitivity $n$, the larger the strain non-uniformity at every moment; at the same time, the larger the ratio $\omega=m / n$, the larger the rate of increase of this strain non-uniformity with time. We note that (16) shows also that, in the absence of non-homogeneities $\left(\omega_{x}(x)=0\right)$, the strain non-uniformities increase at the material regions with initial strain non-uniformities or geometrical defects. Regarding the interplay of $m(x)$ and $n(x)$, we easily verify that combinations of increasing (respectively decreasing) strain rate sensitivity with decreasing (respectively increasing) strain softening, with respect to the spatial position in the material, may lead to high values of $\omega_{x}(x)$ and, by (19), to material zones with intense and increasing non-uniformities. These regions appear, for instance, at the interfaces between different material components, in which the material and thermomechanical parameters are smoothly approximated by the functions $\rho(x)$, $G(x), \sigma(x), m(x)$, and $n(x)$. In the case of composite materials with periodic structure, the strain gradient exhibits a periodic spatial oscillation, which increases monotonically with respect to time.

The same technique can eventually be used to establish the behavior of the strain gradient for the anisothermal case (1). However, in this case, the expression giving $\gamma_{x}$ (corresponding to (16)) contains additional terms involving the temperature gradient with a behavior that cannot easily be defined. So, we restrict ourselves to the numerical 
solution of the anisothermal problem, which is presented in the next section. The numerical results do show, as we will see, that the evolution of the strain gradient is similar to that described above for the isothermal case.

To remedy this situation, we present an alternative analytical description of the interplay between non-homogeneities and non-uniformities, occuring in the case of the anisothermal situation (1) under quasistatic conditions and boundary shear forces (6, first). This description is based on the $L_{\infty}$-localization concept, mentioned in Sec. 1 .

For simplicity, we assume that $\beta \equiv \beta(x)$. The general case can be treated in a similar manner. Under quasistatic conditions, (3) gives

$$
\sigma_{x}(x, t)=0 .
$$

Combining (20) and (6, first), we conclude that

$$
\sigma(x, t)=S
$$

Then, using (4) and (21),

$$
\gamma(x, t)=\rho(x) c(x) \beta(x)^{-1} S^{-1}\left(\theta(x, t)-\theta_{0}(x)\right)+\gamma_{0}(x) .
$$

Using (1), (4), (21), and (22),

$$
\theta(x, t)^{-a(x) / n(x)} \theta_{t}(x, t)(N(x) \theta(x, t)+L(x))^{m(x) / n(x)}=M(x),
$$

where

$$
\begin{gathered}
N(x)=\rho(x) c(x) \beta(x)^{-1} S^{-1}, \quad L(x)=-N(x) \theta_{0}(x)+\gamma(x), \\
M(x)=G(x)^{-1 / n(x)} S^{1 / n(x)} N(x)^{-1} .
\end{gathered}
$$

We easily verify that, if the initial strain is sufficiently large, namely

$$
\gamma_{0}(x)>N(x) \theta_{0}(x), \quad x \in[0, h],
$$

then (23) gives that the temperature is an increasing function of time. Writing (23) at two different points $x_{A}$ and $x_{B}$, and denoting by $\theta_{A}(t)$ and $\theta_{B}(t)$ respectively the temperature at these points, we obtain

$$
\begin{array}{r}
\theta_{A}(t)^{-a_{A} / n_{A}}\left(N_{A} \theta_{A}(t)+L_{A}\right)^{m_{A} / n_{A}} \theta_{A t}(t) \\
=\left(M_{B} / M_{A}\right) \theta_{B}(t)^{-a_{B} / n_{B}}\left(N_{B} \theta_{B}(t)+L_{B}\right)^{m_{B} / n_{B}} \theta_{B t}(t),
\end{array}
$$

where $a_{A}=a\left(x_{A}\right), m_{A}=m\left(x_{A}\right), n_{A}=n\left(x_{A}\right), a_{B}=a\left(x_{B}\right), m_{B}=m\left(x_{B}\right)$, and $n_{B}=n\left(x_{B}\right)$. We assume that

$$
p_{A} / n_{A}<p_{B} / n_{B},
$$

where $p_{A}=m_{A}+n_{A}-a_{A}$ and $p_{B}=m_{B}+n_{B}-a_{B}$, and that the initial temperature at $A, \theta_{0 A}=\theta_{0}\left(x_{A}\right)$, is sufficiently large compared to $\theta_{0 B}=\theta_{0}\left(x_{B}\right)$, namely

$$
\theta_{A}^{p_{A} / n_{A}}>K_{A B} \theta_{0 B}^{p_{B} / n_{B}}
$$

where

$$
K_{A B}=\left(p_{A} n_{B} M_{B} / p_{B} n_{A} M_{A}\right)\left(N_{B}+L_{B} / \theta_{0 B}\right)^{m_{B} / n_{B}} N_{A}^{-m_{A} / n_{A}} .
$$


Then, integration of (25) over time gives

$$
\int \theta_{A}^{\left(m_{A}-\alpha_{A}\right) / n_{A}} d \theta_{A}>\left(M_{B} / M_{A}\right) N_{A}^{-m_{A} / n_{A}} \int \theta_{B}^{-\alpha_{B} / n_{B}}\left(N_{B} \theta_{B}+L_{B}\right)^{m_{B} / n_{B}} d \theta_{B}
$$

and, since $\theta_{B}>\theta_{0 B}$,

$\int \theta_{A}^{\left(m_{A}-n_{A}\right) / n_{A}} d \theta_{A}>\left(M_{B} / M_{A}\right) N_{A}^{-m_{A} / n_{A}} \int \theta_{B}^{-\alpha_{B} / n_{B}}\left(N_{B}+L_{B} / \theta_{0 B}\right)^{m_{B} / n_{B}} \theta_{B}^{m_{B} / n_{B}} d \theta_{B}$

from which, by integrating and recalling (27) and (28),

$$
\theta_{A}^{p_{A} / n_{A}}>K_{A B} \theta_{B}^{p_{B} / n_{B}}
$$

or

$$
\left(\theta_{A} / \theta_{B}\right)^{p_{B} / n_{B}}>K_{A B} \theta_{A}^{\left(p_{B} / n_{B}-p_{A} / n_{A}\right)} .
$$

Since $p_{A} / n_{A}<p_{B} / n_{B}$ (see (26)), the right part of (30) tends to infinity as $t \rightarrow \infty$, the temperature being an increasing function of time.

The analysis presented shows that, even under stability conditions $p(x)=m(x)+$ $n(x)-a(x)>0$, the non-homogeneity of the thermomechanical parameters $p(x)$ and $n(x)$ causes increasing non-uniformity, provided that there exists sufficiently large initial non-uniformity. More specifically, the ratio of $\theta_{A}(t)$ by $\theta_{B}(t)$ increases faster than a power of $\theta_{A}(t)$, as $t \rightarrow \infty$, enhancing the existing non-uniformity and forming always patterned solutions. However, this conclusion is valid only if the initial temperature at $x_{A}$ is sufficiently large compared to the corresponding at $x_{B}$ (see (27) and (28)). Finally, we note that if the material is homogeneous $\left(p_{A}=p_{B}, n_{A}=n_{B}\right)$, the above conclusion is not valid.

3. Numerical Results. We first consider the numerical simulation of thermoviscoplastic shearing of a slab, caused by boundary shear forces, under instability conditions between the thermomechanical parameters. The numerical method chosen to solve this problem of nonlinear partial differential equations (1), (3)-(7), consists of an approximation space in which, for each $t$, the solution vector is approximated by a cubic piece-wise polynomial with $C^{1}$ smoothness (see Houstis (1977)). Then we force the approximate solution to satisfy the system at certain interior (collocation) points, which are chosen according to Houstis (1977). The software implementation was realized by the appropriate selection of parameters of a general finite element collocation program PDECOL (Madsen and Sincovec (1979)). All material parameters used are selected on the basis of a cold-rolled steel-AISI 1018 (see Clifton (1984)), with a constitutive law of the form (1) and with $p=m+n-\alpha<0$. To compare with the non-homogeneous case, we present in Fig. 1 the shear banding of a homogeneous material due to initial geometrical defects. The material is modeled as follows : $G=436 \mathrm{MPa}, \quad \rho=7800 \mathrm{Kg} / \mathrm{m}^{3}, \quad c=$ $500 \mathrm{~J} / \mathrm{Kg} . \mathrm{C}^{0}, \quad m=0.015, \quad n=0.019, \quad$ and $\quad \alpha=0.38$. The strain and strain-rate non-uniformities persist and grow with time, overcoming the action of boundary conditions and causing the collapse of the stress at the middle. We next consider a slab, with $m=0.0145, \quad n=0.0185, \quad$ and $\quad \alpha=0.385$, reinforced by two thin plates exhibiting larger hardening $(m=0.015)$ and strain-rate sensitivity $(n=0.019)$ and smaller softening $(\alpha=0.38)$ (Fig. 2). We have a similar result with the homogeneous case, 
the inhomogeneities being unable to create larger strain gradient at the interfaces. The shear banding appears again at the middle of the slab, where the stress collapses smoothly (Fig. 3). On the contrary, in the case of a larger degree of non-homogeneity, namely $(m, n, \alpha)=(0.018,0.0212,0.37)$ for the plates and $(0.015,0.019,0.38)$ for the slab respectively, the non-homogeneous thermomechanical behavior of the same slab overcomes the action of boundary forces and initial geometrical defects and creates zones with very sharp spatial variation of strain, acting as precursors to failure, at a time smaller than the critical value corresponding to shear banding at the boundaries (Fig. 4). In Fig. 5 we see the strain gradient distribution for various values of time, allowing for the comparison of the strain gradient at the interfaces with the same quantity at the boundaries. The thermal softening coefficient seems to be very important concerning the outcome of the competition between the material non-homogeneities and the action of boundary forces. In Fig. 6 the slab is reinforced by two thin plates with a thermal softening coefficient much closer to the corresponding value of the surrounding material ( $\alpha$ takes the values 0.3705 and 0.38 respectively). This quasi-homogeneous thermal behavior relaxes the non-uniformities at the interior and allows the emergence of localized strain at the boundaries. We now consider a stratified material, made by thin plates with periodic thermomechanical properties, with $(m, n, \alpha)$ oscillating between $(0.015-0.01505,0.019-0.01905,0.38-0.3795)$ respectively (Fig. 7). In Fig. 8 we can see the characteristic oscillation of the strain gradient, indicating that this function converges weakly as the thickness of the layers tends to zero (Charalambakis and Murat 1989 and 1990). In Fig. 9 we can see the collapse of stress at one interface of layers for a time interval of $0.16 \mathrm{msec}$. Similar results are obtained for the case of a non-homogeneous material with thermomechanical parameters satisfying the stability condition (10). In Fig. 10 we can see the distribution of the strain of a slab $(m=0.03, n=0.02, \alpha=0.0202)$ reinforced by two thin plates with considerably higher strength $(m=0.04, n=0.03, \alpha=0.0102)$, while in Figs. 11 and 12 we can see respectively the increasing non-uniformity at the interface and the corresponding collapse of the stress. We close this paragraph by presenting numerical examples for the isothermal case (2). Figs. 13, 14, 15, and 16 depict the behavior of strain, strain gradient, and stress of a slab $(m=0.007, n=0.019)$ reinforced by two thin plates $(m=0.005, n=0.014)$, while Figs. 17 and 18 show the behavior of a composite slab made by thin layers with periodic properties modeled as above. We note that although the above mechanical parameters satisfy the stability conditions (15), the non-uniformities at the interfaces evolve in a practically catastrophic way. We see the non-linearly increasing non-uniformity at the interface, for a time interval of $5 \mathrm{msec}$. The results are in complete agreement with the analytical prediction described in Sec. 2 .

4. Concluding remarks. We have examined the effect of macroscopic non-homogeneities during the simple shearing of thermoviscoplastic materials at high strain rates. Two specific constitutive laws were considered, corresponding to the isothermal and the anisothermal cases.

Stability conditions, based on the concept of bounded strain, were found to be similar with those holding for the homogeneous case. Nevertheless, we have proved that at 
regions where the thermomechanical parameters (strain hardening $m$, strain rate sensitivity $n$, and thermal softening $\alpha$ ) vary considerably with respect to the position, the strain gradient is monotonically increasing in the course of deformation, independently of the relation between strain hardening, strain rate sensitivity, and thermal softening. Then, even stable (in the sense that the blow up of the strain is excluded) solutions may be of catastrophic nature, a conclusion suggesting that new non-uniformity measures, based on the control of the strain gradient, are more suitable to give stability conditions for non-homogeneous materials.

We have presented two aspects of the relation between non-homogeneity and strain non-uniformity: the first is related to the spatial distribution of the strain and the second to its evolution with time. Both of them were found to depend crucially on the degree of non-homogeneity, expressed as functions of the thermomechanical parameters, such as the functions $\omega(x)=m(x) / n(x)$ and $\omega_{x}(x)$ for the isothermal case (see (19)) or the function $p(x) / n(x)$, where $p(x)=m(x)+n(x)-\alpha(x)$, for the anisothermal (see (26), (30)).

Moreover, in the case of a multicomponent material of periodic structure, results similar to those of Charalambakis and Murat (1989 and 1990) were found, showing that the strain gradient oscillates, while the stress converges strongly, as the thickness of the layers becomes very small. It would be of interest to study the complete homogenization problem of thermoviscoplastic shearing for defining the homogenized functions satisfying the system (1)-(7), when the scale parameter tends to zero.

Finally, numerical results were presented concerning the interplay between non-homogeneities, initial defects, and boundary conditions.

\section{Appendix.}

Proposition 1. Assume that $c(x), \rho(x), m(x), n(x), a(x)$, and $\beta\left(x, \gamma, \gamma_{t}, \theta\right)$ are all smooth functions satisfying (10) and (11). Then the solutions of (3)-(7) and (1) satisfy on $[0, h] \times[0, T)$

$$
\sigma(x, t)>0, \quad \gamma_{t}(x, t)>0, \quad \gamma(x, t)>0
$$

and

$$
\sigma(x, t) \leq \Sigma=\max \left(\sup _{x \in[0, h]} \sigma_{0}(x), \sup _{x \in[0, h]}\left(c(x) \rho(x) \beta^{*}(x)^{-1} \theta_{0}(x) \gamma_{0}(x)^{-1}\right)\right) .
$$

Proof. The proof follows the same lines as the corresponding proof in Tzavaras (1987 and 1992) (see also Charalambakis (2001) for the case of strain, strain-rate, and temperature dependent $\beta$ ), the only difference being that the $x$-dependence of the thermomechanical parameters must be taken into account. We present here only a sketch of the proof. We apply the comparison theorem (Protter and Weinberger, 1967, p.187) using 
the reaction-diffusion operator (see (8))

$$
\begin{gathered}
L_{1}[f]=\left(f(x, t)^{1 / n(x)}\right)_{t}-G(x)^{1 / n(x)} \theta(x, t)^{-a(x) / n(x)} \gamma(x, t)^{m(x) / n(x)} \\
\cdot\left(\rho(x)^{-1} f_{x x}(x, t)+(1 / \rho(x))_{x} f_{x}(x, t)\right) \\
-(1 / n(x)) G(x)^{-1 / n(x)} \theta(x, t)^{a(x) / n(x)} \gamma(x, t)^{-m(x) / n(x)} f(x, t)^{2 / n(x)} \\
\cdot\left(-a(x) \rho(x)^{-1} c(x)^{-1} \beta\left(x, \gamma(x, t), \gamma_{t}(x, t), \theta(x, t)\right) f(x, t) \theta(x, t)^{-1}+m(x) \gamma(x, t)^{-1}\right)=0
\end{gathered}
$$

under initial and boundary conditions

$$
f(x, 0)=f_{0}(x)>0, \quad f(0, t)=f(h, t)=S \quad \text { or } \quad f_{x}(0, t)=f_{x}(h, t)=0 .
$$

We first use $f=0$ to conclude that $\sigma(x, t)>0$ and, by virtue of (1) and (4), $\theta(x, t)>$ $\theta_{0}(x)$ and $\gamma(x, t)>\gamma_{0}(x)$, which proves (A.31). Next, we show that, for any $k>\Sigma$, where $\Sigma$ is defined in (A.32), the function $f=k$ satisfies $L_{1}(k)>0$, which, together with $k>\sup _{x \in[0, h]} \sigma_{0}(x), k>\Sigma$ (or, for boundary conditions $\left(6\right.$, second), $\left.f_{x}(0)=f_{x}(h)=0\right)$, yields to $\sigma(x, t)<k$. For the proof, we refer to Charalambakis (2001). We only note that (11) is a sufficient condition for the validity of (A.32).

Proposition 2. The process described by $(3),(4),(5),(1),(6$, first), or $(6$, second) is stable for $t \rightarrow \infty$, provided that inequality (10) holds.

Proof. The proof follows easily from (4) and (A.32): we first write (see (1))

$$
\gamma_{t}(x, t)=G(x)^{1 / n(x)} \sigma(x, t)^{1 / n(x)} \theta(x, t)^{a(x) / n(x)} \gamma(x, t)^{-m(x) / n(x)} .
$$

We next integrate over time (4) and use (A.32) to obtain

$$
\theta(x, t) \leq K \gamma(x, t)
$$

Hence, using (A.36) and (A.32), integrating over time (A.35), and recalling (10), we conclude that we can find a priori bound for $\gamma$ valid for all $t$. In other words, inequality (10) is a sufficient condition for stability, in the sense that the blow-up of the strain at a finite time is excluded.

\section{Appendix.}

Proposition 3. The solutions of (2), (3), (5), (6, first), $\nu(x, 0)=\nu_{0}(x)$ with $\nu_{0 x}(x)>0$, and $\gamma(x, 0)=\gamma_{0}(x)>0$ exhibit the following behavior, as $T \rightarrow \infty$,

$$
\begin{gathered}
\sigma(x, t)=S+O\left(t^{(\lambda-\mu) / 2}\right), \\
\gamma(x, t)^{(n(x)-m(x)) / n(x)}=S t+O\left(t^{(\lambda-\mu) / 2}\right),
\end{gathered}
$$

where

$$
\lambda=\sup _{x \in[0, h]}(m(x) /(n(x)-m(x))), \quad \mu=\inf _{x \in[0, h]}((2 n(x)-3 m(x)) /(n(x)-m(x))) .
$$


Proof. The proof follows the same lines as Tzavaras $(1987,1991)$. The first step is to show that

$$
\phi(x) \leq \sigma(x, t) \leq \sup _{x \in[0, h]} \sigma_{0}(x)
$$

where

$$
\phi(x)=\alpha\left(\int_{0}^{x} \rho(\xi) \xi \mathrm{d} \xi+1\right)
$$

with the positive constant $\alpha$ being bounded by

$$
\begin{aligned}
& \alpha^{(2-n(x)) / n(x)}<\min \left(\inf _{x \in[0, h]}\left(\sigma_{0}(x) /\left(\int_{0}^{x} \rho(\xi) \xi \mathrm{d} \xi+1\right)\right)\right. \\
& \left.1 / \sup _{x \in[0, h]}\left(n(x)^{-1} m(x) G(x)^{-2 / n(x)} \gamma_{0}(x)^{(2 m(x)-n(x)) / n(x)}\left(\int_{0}^{x} \rho(\xi) \xi \mathrm{d} \xi+1\right)^{2 / n(x)}\right)\right)
\end{aligned}
$$

This can be easily obtained by applying comparison principles (Protter and Weinberger, 1967, p.187) in the differential operator

$$
\begin{aligned}
& L_{2}[f]=f_{t}(x, t)-n(x) G(x)^{1 / n(x)} f^{(n(x)-1) / n(x)} \gamma^{-m(x) / n(x)} \\
& \quad\left(\rho(x)^{-1} f_{x}(x, t)\right)_{x}+m(x) G(x)^{-1 / n(x)} f^{(1+n(x)) / n(x)} \gamma(x, t)^{(m(x)-n(x)) / n(x),}
\end{aligned}
$$

which can be shown to be uniformly parabolic and satisfies $L_{2}[\sigma]=0$ (see (2)). The function $\phi(x)$ is defined by imposing $L_{2}[\phi]<0$ and $\phi(x) \leq \sigma_{0}(x), \quad \forall \quad x \in[0, h]$. Then, recalling (14),

$$
K^{-1} t \leq \gamma(x, t)^{(m(x)-n(x)) / n(x)} \leq K t .
$$

In view of (3) and (6, first) we have the following identity:

$$
\frac{1}{2} \frac{\mathrm{d}}{\mathrm{d} t} \int_{0}^{h} \rho(x) \nu_{t}(x, t)^{2} \mathrm{~d} x+\int_{0}^{h} \sigma_{t}(x, t) \nu_{x t}(x, t) \mathrm{d} x=0,
$$

or, by (2),

$$
\begin{aligned}
& \frac{1}{2} \frac{\mathrm{d}}{\mathrm{d} t} \int_{0}^{h} \rho(x) \nu_{t}(x, t)^{2} \mathrm{~d} x \\
& \quad+\int_{0}^{h} n(x) G(x)^{1 / n(x)} \gamma(x, t)^{-m(x) / n(x)} \sigma(x, t)^{(n(x)-1 / n(x))} \nu_{x t}(x, t)^{2} \mathrm{~d} x \\
& =\int_{0}^{h} m(x) G(x)^{-1 / n(x)} \gamma(x, t)^{(m(x)-n(x)) / n(x)} \sigma(x, t)^{(1+n(x)) / n(x)} \nu_{t}(x, t) \mathrm{d} x .
\end{aligned}
$$

By virtue of the boundary conditions $\left(6\right.$, first), there exists $x_{0} \in[0, h]$ such that

$$
\nu_{t}\left(x_{0}, t\right)=\sigma_{x}\left(x_{0}, t\right)=0, \quad \forall \quad t \in[0, T),
$$

which, together with Schwarz's inequality, lead to

$$
\nu_{t}(x, t)^{2} \leq K \int_{0}^{h} \nu_{x t}(x, t)^{2} \mathrm{~d} x
$$


Using (B.45) and Schwarz's inequality,

$$
\begin{aligned}
\frac{\mathrm{d}}{\mathrm{d} t} \int_{0}^{h} \rho(x) \nu_{t}(x, t)^{2} \mathrm{~d} x+ & K^{-1} \int_{0}^{h} \gamma(x, t)^{-m(x) / n(x)} \sigma(x, t)^{(n(x)-1) / n(x)} \nu_{x t}(x, t)^{2} \mathrm{~d} x \\
& \leq K \int_{0}^{h} \gamma(x, t)^{(3 m(x)-2 n(x)) / n(x)} \sigma(x, t)^{(3+n(x)) / n(x)} \mathrm{d} x .
\end{aligned}
$$

Combining (B.40), (B.44), (B.46), and (B.39) with (B.47), we arrive at the differential inequality

$$
\mathrm{d} \psi(t) / \mathrm{d} t+K^{-1} t^{-\lambda} \psi(t) \leq K t^{-\mu}
$$

where

$$
\psi(t) \equiv \int_{0}^{h} \nu_{t}(x, t)^{2} \mathrm{~d} x .
$$

Integrating (B.48) and taking into account that $m(x)<n(x) / 2$, we obtain

$$
\int_{0}^{h} \nu_{t}(x, t)^{2} \mathrm{~d} x \leq K t^{-\mu+\lambda}
$$

from which, using $(\sigma(x, t)-S)^{2} \leq K \int_{0}^{h} \sigma_{x}(x, t)^{2} \mathrm{~d} x \leq K \int_{0}^{h} \nu_{t}(x, t)^{2} \mathrm{~d} x$, we obtain (B.37). Recalling (14) and using (B.37), we easily obtain (B.38).

We note that, to establish the above results, we only need the weaker assumption $m(x)<n(x) / 2$. However, in order to more easily study the behavior of the strain gradient, we assume that $m(x)<n(x) / 3$. Recalling (B.47), (B.37), and (B.38),

$$
\mathrm{d} \psi(t) / \mathrm{d} t+K^{-1} t^{-\lambda} \int_{0}^{h} \nu_{x t}^{2} \mathrm{~d} x \leq K t^{-\mu}
$$

from which, by integration,

$$
\int_{0}^{t} \int_{0}^{h} \nu_{x t}(x, \tau)^{2} \mathrm{~d} x \mathrm{~d} \tau \leq K t^{1-\mu+\lambda} .
$$

We note that, since $m(x)<n(x) / 3$, then $1-\mu+\lambda<0$. The estimate (B.52) is necessary to obtain the asymptotic behavior of the strain gradient (see (16), (17), (18)).

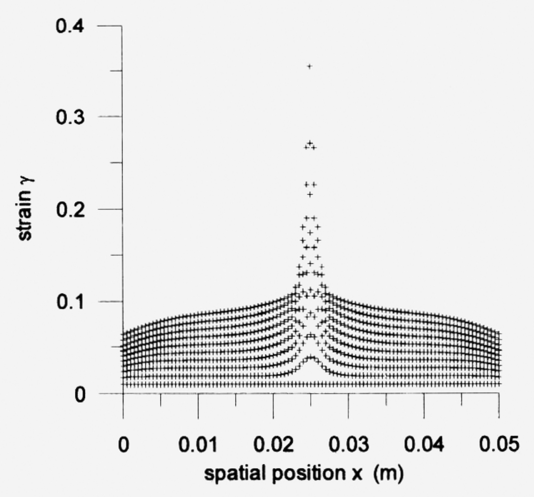

FIG. 1. Shear banding of homogeneous slab. 


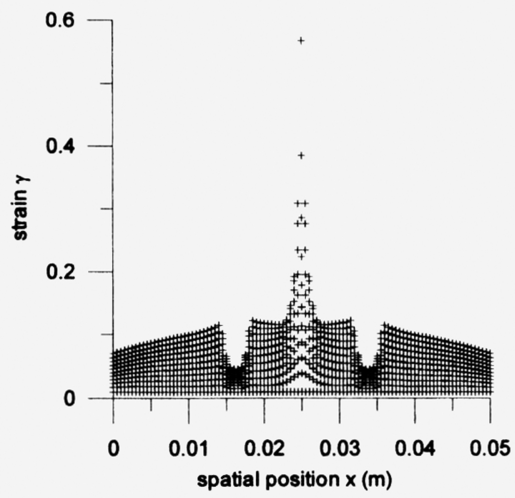

FIG. 2. Strain of reinforced slab.

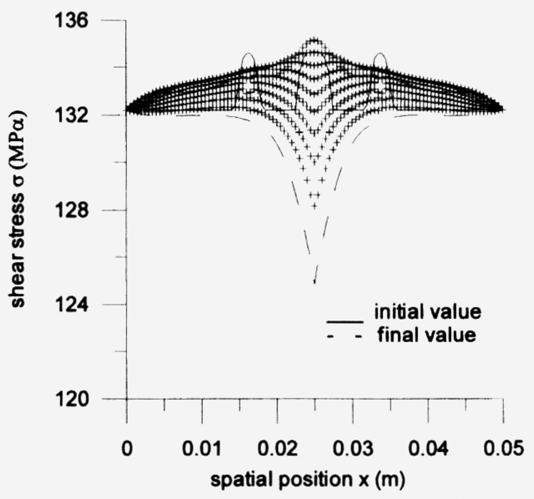

FIG. 3. Evolution of stress distribution.

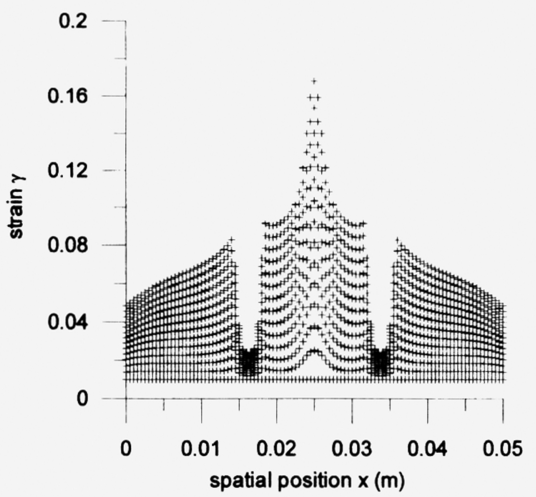

FIG. 4. Reinforced slab. Large non-homogeneity overcomes the action of boundary forces and initial defects. 


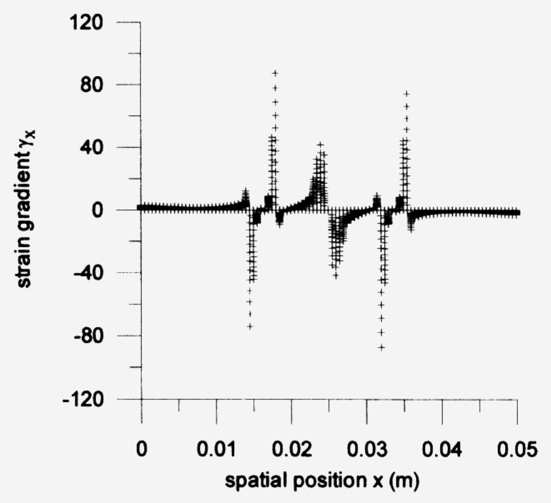

FIG. 5. Reinforced slab. Strain gradient distribution.

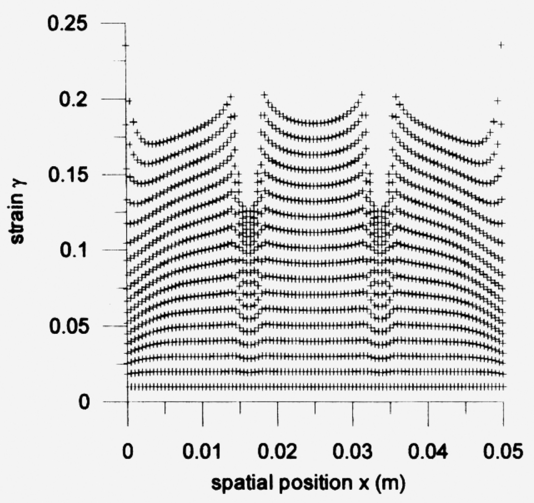

FIG. 6. Quasi-homogeneous thermal behavior.

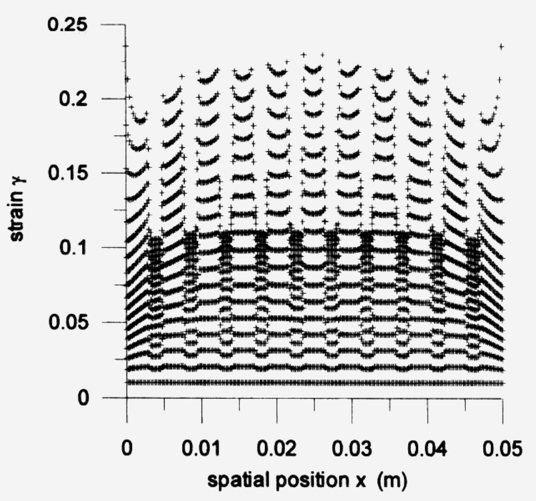

FIG. 7. Stratified slab with periodic structure. 


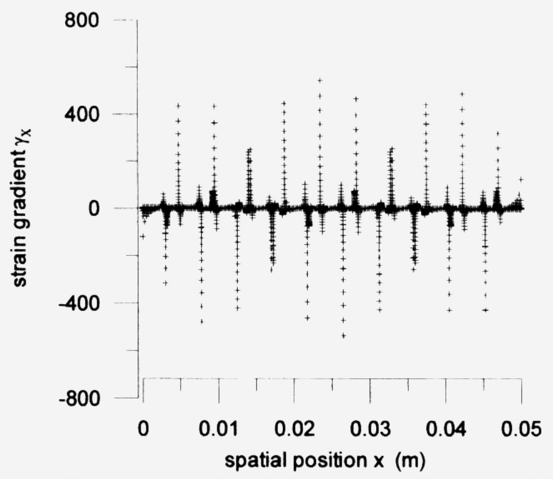

FIG. 8. Oscillation of the strain gradient.

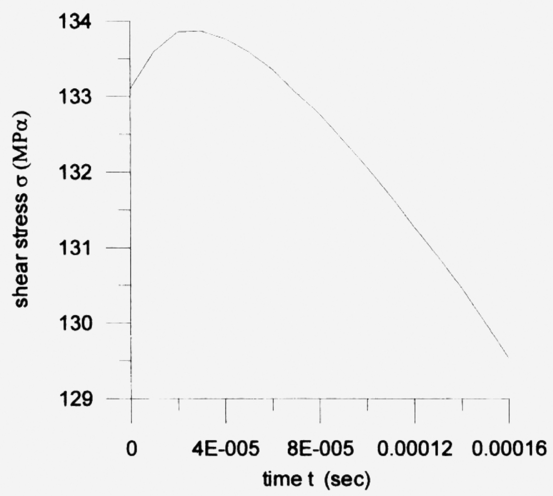

FIG. 9. Collapse of stress at interface of layers.

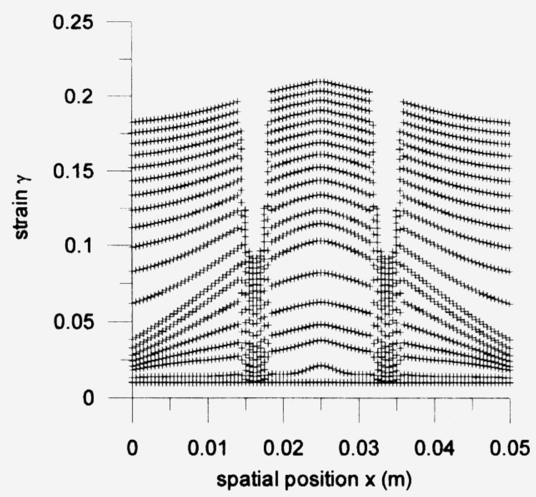

FIG. 10. Strain of reinforced slab (case of stability $p(x)>0, \forall x$ ). 


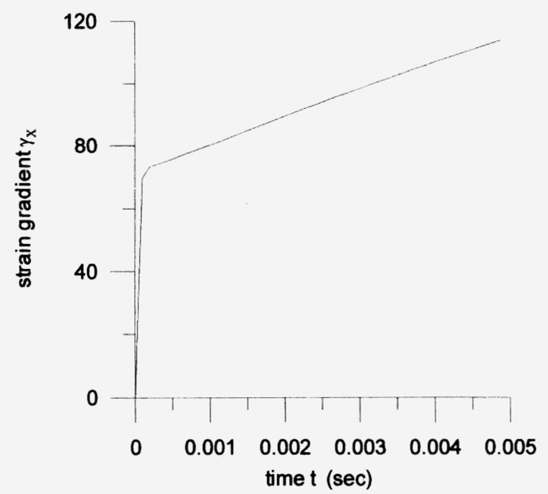

FIG. 11. Increase of strain gradient at an interface (case of stability $p(x)>0, \forall x)$.

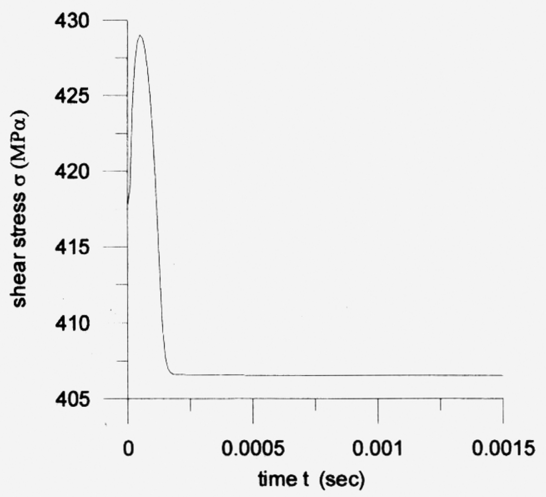

FIG. 12. Collapse of stress at an interface (case of stability $p(x)>$ $0, \forall x)$.

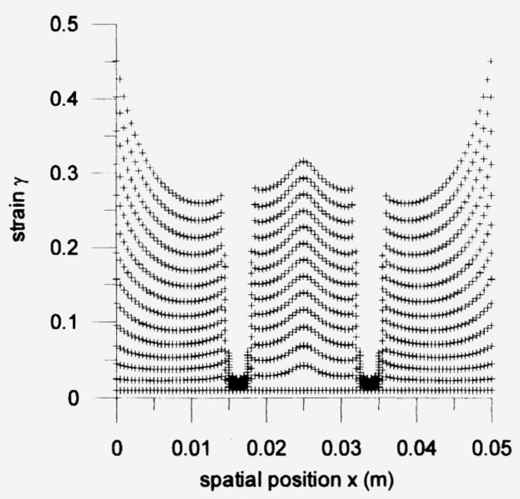

FIG. 13. Isothermal case. Strain of reinforced slab. 


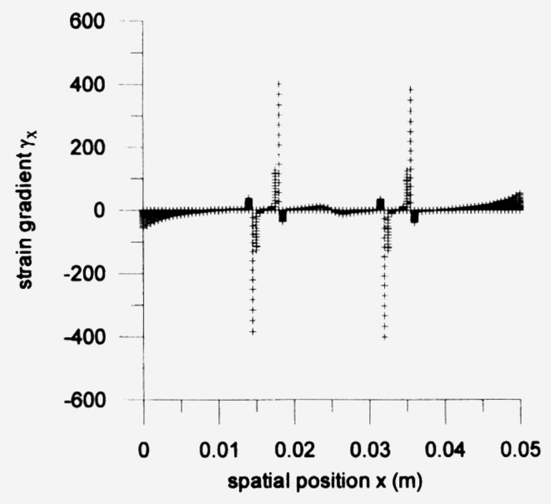

FIG. 14. Isothermal case. Strain gradient of reinforced slab.

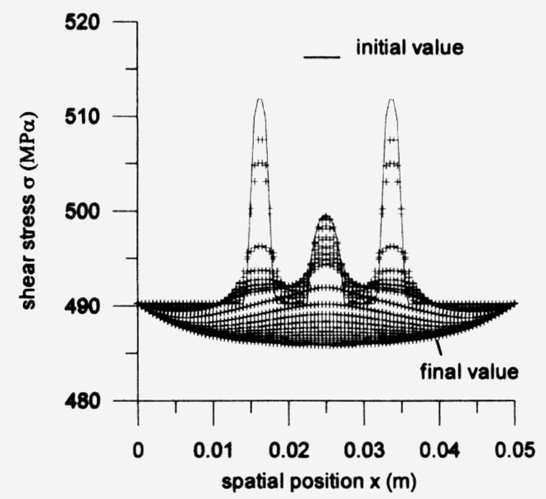

FIG. 15. Isothermal case. Stress of reinforced slab.

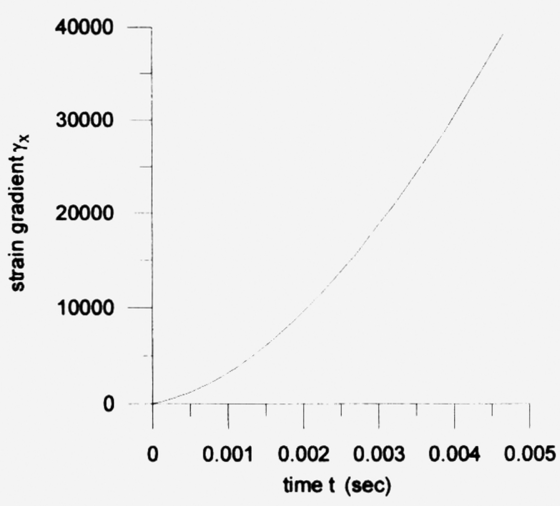

FIG. 16. Isothermal case. Increase of strain gradient at an interface. 


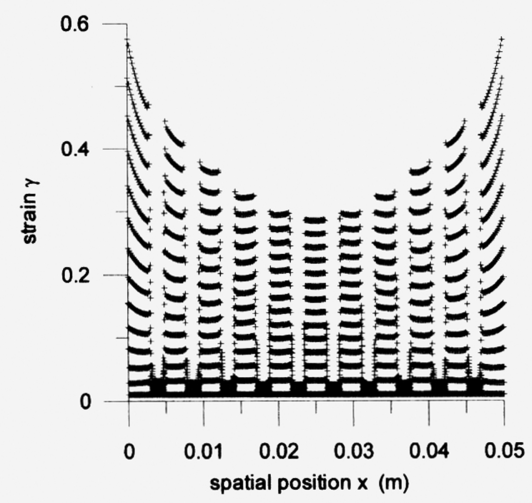

FIG. 17. Isothermal case. Strain of multicomponent plate.

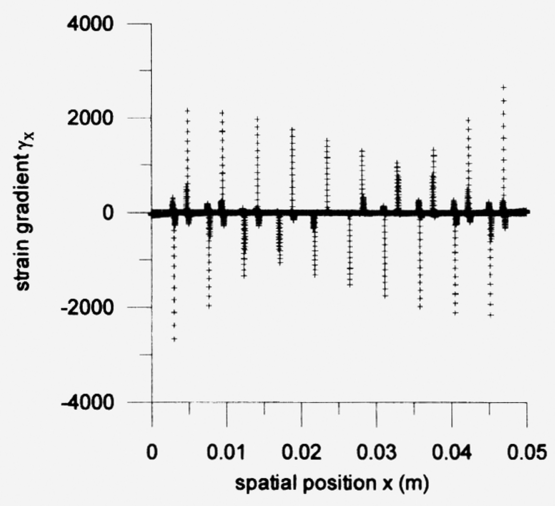

FIG. 18. Isothermal case. Oscillation of strain gradient.

\section{REFERENCES}

Bai, Y., (1982) Thermoplastic instability in simple shear. J. Mech. Phys. Solids 30, pp $195-207$.

Bensoussan, A., Lions, J.L., Papanikolaou, G., 1978. Asymptotic Analysis for Periodic Structures, North Holland.

Burns, T., (1983) SAND83-1907, Sandia National Laboratories, UC-32.

Charalambakis, N., (1984) Adiabatic shearing flow caused by time dependent inertial force. Quart. Appl. Math. 42, pp 275 - 280.

Charalambakis, N., (1985) Time-asymptotic stability of non-Newtonian fluid or plastic solid. Mech. Research Com. 12, pp 311 - 317.

Charalambakis, N. and Murat, F., (1989) Weak solutions to the initial-boundary problem for the shearing of non-homogeneous thermoviscoplastic materials. Proc. Royal Soc. Edinburgh 113A, pp 257 -265 .

Charalambakis, N. and Murat, F., (1990) Homogenization of stratified thermoviscoplastic materials, unpublished.

Charalambakis, N., (2001) Shear stability and strain, strain-rate and temperature dependent cold work. Int. J. Eng. Sci. 39, pp 1899 - 1911.

Chen, H.T., Douglas, A.S., and Malek-Madani, R., (1989) An asymptotic stability condition for inhomogeneous simple shear. Quart. Appl. Math. 47, pp 247 - 262. 
Clifton, R.J., Duffy, J., Hartley, K.A., and Shawki, T.G., (1984) On critical conditions for shear band formation at high strain-rates. Scripta Met. 18, pp $443-448$.

Dafermos, C.M., (1982) Global smooth solutions to the initial boundary value problem of one dimensional nonlinear thermoviscoelasticity. SIAM J. Math. Analysis 13, pp $397-408$.

Dafermos, C.M. and Hsiao, L., (1983) Adiabatic shearing of incompressible fluids with temperature dependent viscosity. Quart. Appl. Math 41, pp 45 - 58.

Dafermos, C.M., (1985) Contemporary Issues in the Dynamic Behavior of Continuous Media, LCDS Lecture Notes $\sharp 85-1$.

Dodd, B. and Bai, Y., (1987) Ductile Fracture and Ductility, Academic Press.

Fressengeas, C. and Molinari, A., (1987) Instability and localization of plastic flow in shear at high strain rates. J. Mech. Phys. Solids 35, pp 185 - 211.

Hodowany, J., Ravichandran, G., Rosakis, A.J., and Rosakis, P., (2000) Partition of plastic work into heat and stored energy in metals. J. Exper. Mech. 40, pp $113-123$.

Houstis, E. N., (1977) Application of method of collocation on lines for solving hyperbolic systems, Math. Comp. 31, pp 443 - 456.

Leroy, Y. and Molinari, A., (1992) Stability of steady states in shear zones. J. Mech. Phys. Solids 40, pp $181-212$.

Liu, Q. and Jiang, S., (2003) Global existence and numerical approximation in adiabatic shearing of incompressible fluids. J. Comput. Appl. Math. 154(2), pp. 303-317.

Madsen, N. K. and Sincovec, R. F., (1979) PDECOL : General collocation software for PDE, ACN. Trans. Math. Software 5.

Maddoks, J.H. and Malek-Madani, R., (1992) Steady-state shear-bands in thermoplasticity, Part I: Vanishing yield stress. Int. J. Solids Structures 29, pp 2039 - 2061.

Molinari, A., (1985) Instabilite thermoviscoplastique en cisaillement simple. J. Mec. Theor. Appl. 4, pp $659-684$.

Molinari, A. and Clifton, R.J., (1987) Analytical characterization of shear localization in thermoviscoplastic materials. Journal of Applied Mechanics 54, pp 806 - 812.

Protter, M.H. and Weinberger, H.F., (1967) Maximum Principles in Differential Equations, Prentice Hall, Englewood Cliffs, New Jersey.

Rigatos, A. and Charalambakis, N., (2001) Two-dimensional adiabatic newtonian flow with temperature dependent viscosity. Int. J. Eng. Sci. 39/10, pp 1143 - 1165.

Rosakis, P., Rosakis, A.J., Ravichandran, G., and Hodowany, J., (2000) A thermodynamic internal variable model for the partition of plastic work into heat and stored energy in metals. J. Mech. Phys. Solids 48, pp 582 - 607.

Shawki, T.G. and Clifton, R.J., (1989) Shear band formation in thermal viscoplastic materials. Mech. Materials 8, pp 13 - 43.

Suquet, P., (1982) Plasticite et homogeneisation, These de Doctorat dÉtat, Univ. Pierre et Marie Curie, Paris.

Tzavaras, A.E., (1986) Plastic shearing of materials exhibiting strain hardening or strain softening. Arch. Rat. Mech. Anal. 94, pp 39 - 58.

Tzavaras, A.E., (1987) Effect of thermal softening in shearing of strain-rate dependent materials. Arch. Rat. Mech. Anal. 99, pp 349 - 374.f

Tzavaras, A.E., (1991) Strain softening in viscoelasticity of the rate type. J. Integr. Eq. Appl. 3, pp $195-238$.

Tzavaras, A.E., (1992) Nonlinear analysis techniques for shear band formation at high strain rates. Appl. Mech. Reviews 45, pp 82 - 94.

Wright, T.W. and Batra, R.C., (1985) The initiation and growth of adiabatic shear bands. Int. J. Plasticity 1, pp $205-212$.

Wright, T.W. and Walter, J.W., (1987) On stress collapse in adiabatic shear bands. J. Mech. Phys. Solids 35, pp 701 - 720. 